\title{
Research Square \\ HOXB9 Promotes Laryngeal Squamous Cell Carcinoma Progression by Upregulating MMP12
}

\section{Chuanhui Sun}

The first Affiliated Hospital of Guizhou University of Traditional Chinese Medicine

\section{Peng Wang}

The Second Affiliated Hospital of Harbin Medical University

\section{Yujiang Chen}

The first Affiliated Hospital of Guizhou University of Traditional Chinese Medicine

\section{Qiuying Li}

The Second Affiliated Hospital of Harbin Medical University

\section{Hua Deng}

The first Affiliated Hospital of Guizhou University of Traditional Chinese Medicine

Changsong Han ( $\square$ han050122@163.com )

The First Affiliated Hospital of Guizhou University of Traditional Chinese Medicine https://orcid.org/0000-0001-7938-6740

\section{Yanan Sun}

The Second Affiliated Hospital of Harbin Medical University

\section{Research}

Keywords: Laryngeal squamous cell cancer, HOXB9, CRISPR/CAS9, MMP12

Posted Date: June 7th, 2021

DOI: https://doi.org/10.21203/rs.3.rs-554245/v1

License: (c) (1) This work is licensed under a Creative Commons Attribution 4.0 International License. Read Full License 


\section{HOXB9 promotes laryngeal squamous cell carcinoma progression by}

\section{upregulating MMP12}

Chuanhui Sun ${ }^{1, \#}$, Peng Wang ${ }^{2, \#}$, Yujiang Chen ${ }^{3}$, Qiuying $\mathrm{Li}^{2}$, Hua Deng ${ }^{1}$, Yanan Sun ${ }^{2, *}$, Changsong $\operatorname{Han}^{3, *}$

${ }^{1}$ Department of Otorhinolaryngology, Head and Neck Surgery, The First Affiliated Hospital of Guizhou University of Traditional Chinese Medicine, Guiyang, PR China.

${ }^{2}$ Department of Otorhinolaryngology, Head and Neck Surgery, The Second Affiliated Hospital, Harbin Medical University, Harbin, PR China.

${ }^{3}$ Department of Pathology, The First Affiliated Hospital of Guizhou University of Traditional Chinese Medicine, Guiyang, PR China.

*Correspondence to: Yanan Sun, Department of Otorhinolaryngology, Head and Neck Surgery, The Second Affiliated Hospital, Harbin Medical University, Harbin150001, PR China, Phone number: 86-451-86605222, E-mail:yanansun2012@163.com; Changsong Han, Department of Pathology, The First Affiliated Hospital of Guizhou University of Traditional Chinese Medicine, Guiyang550001, PR China, Phone number: 86-851-85609789, E-mail: han050122@163.com.

\# Chuanhui Sun and Peng Wang contributed equally to this work. 


\section{ABSTRACT}

Background: The HOX family transcription factor HOXB9 is a crucial element in the progression of various cancers. In the previous study conducted by the investigators, a drastically higher HOXB9 expression was reported in laryngeal squamous cell cancer (LSCC), when compared to adjacent normal laryngeal squamous tissues. Furthermore, a high level of HOXB9 was closely correlated with histological grade and overall survival in LSCC patients. However, the underlying molecular mechanisms have not been fully elucidated.

Results: The present study explored the molecular mechanisms of HOXB9 in LSCC progression. Furthermore, the in vitro and in vivo studies revealed that the gene knockout of HOXB9 using the CRISPR/CAS9 system inhibited cell proliferation, migration and invasion, and promoted cell apoptosis. Mechanistic studies in LSCC cell lines and human LSCC specimens demonstrated that HOXB9 promotes LSCC progression by directly upregulating the MMP12 expression at the level of its transcription.

Conclusions: Collectively, the present study is the first to demonstrate the role of HOXB9 in the regulation of LSCC progression by enhancing the upregulation of MMP12.

Keywords: Laryngeal squamous cell cancer, HOXB9, CRISPR/CAS9, MMP12

\section{INTRODUCTION}

Among all head and neck cancers, laryngeal squamous cell carcinoma (LSCC) remains as the second most common malignant squamous cell carcinoma. ${ }^{1}$ At present, the treatment of LSCC is mainly traditional surgical treatment supplemented by radiotherapy and chemotherapy. ${ }^{2}$ However, there are many side effects and limited 
therapeutic effects with its long-term use. At present, a deep understanding of the underlying molecular mechanisms that promote LSCC remains elusive, and there is a lack of effective markers and related targets for the diagnosis and treatment of LSCC. Therefore, it is of great significance to actively explore the key molecules involved in the occurrence and development of LSCC, in order to improve the treatment effect and survival rate of patients.

The HOX gene has been reported to play an important role in human embryonic development and cell differentiation. ${ }^{3}$ According to the similarity of the human HOX gene sequence and chromosome location, the HOX gene can be divided into four clusters (HOXA-D), ${ }^{4,5}$ and each cluster has 13 paralogous groups. ${ }^{6,7}$ Aberrations in HOX gene expression often leads to abnormal development and malignant tumors. At present, a large number of literatures have reported that the HOX gene is involved in the occurrence and development of different tumors, such as breast cancer, leukemia, lung cancer and gastric cancer. ${ }^{8-13}$ HOXB9 is one of the important members of the HOX family. As a transcription factor, this plays an important role in embryonic development and cancer progression. ${ }^{14-16}$

There are few reports on the HOX gene and LSCC. At present, there is no large sample microarray and histological detection of the HOX gene in LSCC. In the previous study conducted by the investigators, it was found that the expression of HOXB9 is higher in LSCC than in paracancerous tissues. In addition, a high level of HOXB9 is notably correlated with the high histological grade and poor prognosis of LSCC patients. $^{17}$

However, the mechanism underlying HOXB9 in LSCC progression remains unknown. The present study explored the role of HOXB9 in the proliferation, 
migration, invasion and apoptosis of LSCC cells. In addition, the present data revealed that MMP12 is an important target gene regulated in the downstream of HOXB9. Importantly, HOXB9 promoted LSCC progression by upregulating the MMP12 protein expression in LSCC cells via direct binding to the promoter of MMP12.

\section{MATERIALS AND METHODS}

\section{Cell culture}

Human LSCC cell lines (Hep-2 and AMC-HN-8) and a normal human keratinocyte cell line (HaCaT) were obtained from the Shanghai Institute of Biochemistry and Cell Biology (Shanghai, China). All cells were cultured in Dulbecco's modified Eagle's medium (DMEM) supplemented by $10 \%$ fetal bovine serum (FBS; Gibco, Carlsbad, CA, USA), and cultured at $37^{\circ} \mathrm{C}$ with $5 \% \mathrm{CO}_{2}$.

\section{CRISPR/cas9-mediated knockout of HOXB9 in LSCC cells}

The CRISPR/Cas9 system was essentially used to target HOXB9. LSCC cell lines, including AMC-HN-8 and Hep-2 cells, were transfected with single-guide RNA (sgRNA) for three independent specific sequences of HOXB9 or nonspecific sgRNA. These were designed using the online CRISPR design (http://crispr.mit.edu/). After 48 hours of infection, AMC-HN-8 and Hep-2 cells were screened with $1 \mu \mathrm{g} / \mathrm{ml}$ of puromycin for seven days to generate stably transfected cells. Then, these selected cells were expanded in regular culture medium (DMEM medium supplemented with 10\% FBS). The HOXB9 protein expression levels in sgRNA-transfected or control cells were confirmed by western blot. 


\section{The reverse transcription-quantitative polymerase chain reaction (RT-qPCR)}

assay

The total RNA were extracted from different cell lines using Trizol reagent (Invitrogen, Carlsbad, CA, USA), according to manufacturer's instructions. Approximately $2 \mu \mathrm{g}$ of total RNA sample was reverse transcribed into cDNA using a high capacity cDNA kit (Thermo Fisher Scientific, Waltham, MA, USA). The relative mRNA levels were normalized against GAPDH, and calculated using the $2^{-\Delta \Delta C t}$ method, all measurements were performed in triplicate.

\section{Western blot analysis}

The total protein obtained from the different cell lines $(20 \mu \mathrm{g})$ were separated on $10 \%$ sodium dodecyl-sulfate polyacrylamide gel electrophoresis (SDS-PAGE) and transferred onto a polyvinylidene fluoride (PVDF) membrane. After blocking with 5\% bovine serum albumin (BSA) at $25^{\circ} \mathrm{C}$ for one hour, the membrane was incubated overnight at $4^{\circ} \mathrm{C}$ with different primary target protein antibodies or GAPDH. After incubating with the HRP-conjugated IgG antibody (1:8,000 dilution; Zhongshan Jinqiao Technology, Beijing, China) at $37^{\circ} \mathrm{C}$ for one hour, the proteins were visualized using an enhanced ECL Kit (Thermo Fisher Scientific, Waltham, MA, USA). GAPDH was used as a loading control, and the bands were quantified using the Quantity One Software v4.62 (Bio-Rad, Hercules, CA, USA). All measurements were performed in triplicate.

\section{Cell proliferation assay}

The role of HOXB9 on LSCC cell proliferation was evaluated using Cell Counting Kit-8 (CCK-8) assay (Beyotime Biotechnology; Shanghai, China), 
according to manufacturer's instructions. Briefly, Hep-2 and AMC-HN-8 cells were added to $96-$ well plates at a concentration of $3 \times 10^{3}$ cells per well. A total of $10 \mu 1$ of CCK-8 solution was added for one hour into each well at different time points $(24,48$, 72 and 96 hours), and the absorbance at $450 \mathrm{~nm}$ was measured using an enzyme-linked immunosorbent assay (ELISA) reader (Bio-Rad Laboratories, Inc., Hercules, CA, USA). The results were representative of three individual experiments performed in triplicate.

\section{Cell cycle analysis}

The different cell cycles were measured according to manufacturer's instructions (BD Biosciences, CA, USA). These were performed using flow cytometry, as previously described. ${ }^{18}$ All experiments were repeated for at least three times.

\section{Cell migration assay}

In order to evaluate the changes in migration ability after the knockout HOXB9, wound healing and Transwell assay were separately performed. Briefly, Hep-2 and AMC-HN-8 cells were seeded on six-well plates, and grown up to 80-90\% confluence. Then, the cell monolayer was wounded using a $200-\mu 1$ plastic tip, and the wounds were observed at different time points ( 0,12 and 24 hours post-wounding) within the scrape line. The images were captured using a microscope, and the migration ability was analyzed by measuring the width of the wounds. In the Transwell assay, the cell suspension that contained $5 \times 10^{4}$ cells were seeded into the upper chamber $(8 \mu \mathrm{m}$ in size) of a 24 -well plate in serum-free media. The lower wells contained $10 \%$ FBS. After incubation for 24 hours, cells that passed through the inserts were fixed in $4 \%$ methanol for 20 minutes, and stained by $0.1 \%$ crystal violet. Then, the migrated cells 
were counted under a microscope, and images of three random fields were scanned. These results were representative of three individual experiments.

\section{Cell invasion assay}

The Transwell champers were coated with $80 \mu \mathrm{l}$ of Matrigel (BD Biosciences, CA, USA) on a 24 -well plate. Different cells $\left(5 \times 10^{4}\right)$ were added to the upper Transwell chambers in serum-free media, while the lower wells contained 10\% FBS. After incubation for 24 hours at $37^{\circ} \mathrm{C}$, cells that invaded on the lower membrane were fixed with methanol and stained with $0.1 \%$ crystal violet for 20 minutes. The number of invading cells were counted under a microscope, and the images of three random fields were captured. All measurements were repeated in triplicate.

\section{TUNEL assay}

The apoptosis of cells was detected using a TUNEL detection kit (Roche), according to manufacturer's instructions. Hep-2 and AMC-HN-8 cells $\left(10^{4} / \mathrm{ml}\right)$ were inoculated on the cover glass of the 24-well plate, and cultured for 24 hours. Then, cells were fixed with $4 \%$ paraformaldehyde at room temperature for 20 minutes, and incubated with $0.1 \%$ Triton $\mathrm{X}-100$ on ice for two minutes. Afterwards, the TUNEL reagent was added and incubated at $37^{\circ} \mathrm{C}$ for 60 minutes, and cells were dyed with DAPI for five minutes, and observed under a microscope.

\section{In vivo tumorigenicity assay}

Ten BALB/c nude mice (5 weeks of age), which were obtained from Vital River Laboratories (Beijing, China), were housed in aseptic conditions. This animal experiment was approved by the Ethics Committee of Harbin Medical University. All 
mice were subcutaneously injected into the flank with $1 \times 10^{6}$ suspension of Hep-2 cells (150 $\mu \mathrm{l}$ of DMEM/Matrigel at 1:1 mixture). Then, the tumor sizes were measured every three days after becoming palpable under the skin. These tumor-bearing mice were sacrificed at four weeks when the tumor reached approximately $1.5 \mathrm{~cm}$ in diameter. These tumors were removed for weighing and further study.

\section{Affymetrix microarray analysis}

Six RNA samples (three from HOXB9-knockout cells and three from control sgRNA-transfected Hep-2 cells) were used for the microarray test. The total RNA (100 mg) was isolated using TRIzol reagent (Invitrogen, Carlsbad, CA, USA), according to manufacturer's instructions. These microarray experiments were carried out, as we previously described. ${ }^{17}$ The microarray data were analyzed using normalized transcript signals. Hierarchical clustering was applied to visualize the expression of all differential genes, the differences in gene expression between HOXB9-knockout and control sgRNA-transfected Hep-2 cell samples were calculated, and those that were greater than 2-fold upregulated/downregulated were considered significant.

\section{Immunohistochemistry analysis}

The samples were fixed in $4 \%$ formalin and paraffin-embedded. Immunohistochemistry was performed, as previously described. ${ }^{17}$ The slides were incubated with the primary antibody against HOXB9 (1:200; Abcam, Cambridge, MA, USA) and MMP12 (1:400; Abcam, Cambridge, MA, USA) overnight at $4^{\circ} \mathrm{C}$. The sections were developed using diaminobenzidine (DAB), and all histological 
assessments were conducted by two experienced pathologists. The score of the immunohistochemical staining was calculated by multiplying the staining intensity with the stained area of positive cells. Staining intensity score: 0 for negative, 1 for low staining intensity, 2 for medium staining intensity, and 3 for high staining intensity. The staining area of positive cells was scored, as follows: 0 , none; $1,<10 \%$; $2,10-50 \% ; 3,>50 \%$. A total score of 4 was used to distinguish between low $(<4)$ and high $(\geq 4)$ levels of HOXB9 and MMP12 gene expression.

\section{Luciferase assay}

The target cells (Hep-2 cells with or without HOXB9-knockout and HEK-293T cells transfected with pcDNA3.1 or pcDNA3.1-overexpressed HOXB9) were separately transfected with wild-type or mutant MMP12 luciferase reporter plasmids, and the inner control was the luciferase reporter gene. All cells in the logarithmic growth stage were digested with trypsin to make the cell suspension. After 48 hours of transfection, $100 \mu \mathrm{l}$ of lysate was added into each pore and centrifuged at $12,000 \times \mathrm{g}$ for five minutes, and the supernatant was collected for determination. The activities of the firefly and Renilla luciferases were determined using the Dual-Glo Luciferase Assay System (E1960, Promega), according to manufacturer's instructions. The normalized data were calculated as the ratio of Renilla/firefly luciferase activities.

\section{Chromatin immunoprecipitation (ChIP) assays}

The HEK-293T and Hep-2 cell lines $\left(1 \times 10^{7}\right)$ were crosslinked with $1 \%$ formaldehyde for 10 minutes on ice, and subjected to ChIP assays using a specific antibody, according to manufacturer's protocol (Millipore). The ChIP DNA complex was precipitated and subjected to RT-qPCR using MMP12 sense primer F 
5'-CATGAACCGTGAGGATGTTGA-3' and antisense primer R 5'GCATGGGCTAGGATTCCACC-3’.

\section{RESULTS}

The expression of HOXB9 in LSCC cell lines and the human normal keratinocyte line

In order to determine the expression of HOXB9 in the LSCC cell line, the mRNA expression of HOXB9 in the human normal keratinocyte line (HaCaT) and LSCC cell lines (Hep-2 and AMC-HN-8) was detected by RT-qPCR. The results revealed that the mRNA expression of HOXB9 in the LSCC cell lines were higher than that in HaCaT (Figure 1A). The protein expression of HOXB9 was detected by western blot, and it was revealed that the expression of HOXB9 in the LSCC cell lines were higher than that in the human keratinocyte HaCaT (Figure.1B).

\section{Efficiency verification of HOXB9 knockout in LSCC cells}

Three different HOXB9 knockout LSCC cells (KO-1, KO-2 and KO-3), and cells in the NC group and control group were used to verify the knockout of HOXB9 by immunoblotting. The protein expression levels of HOXB9 in Hep-2 and AMC-HN-8 significantly disappeared at 72 hours after KO-3 knockout (Figure 2). Therefore, the KO-3 of HOXB9 was selected and collected as a stable knockout HOXB9 cell line for the subsequent cellular functional experiments.

\section{HOXB9 affects the cellular functional changes of LSCC cells}

Given the high expression level of HOXB9 in LSCC, the effect of HOXB9 on the functional changes of LSCC cells was investigated. The CCK-8 assay was initially 
used to investigate the proliferation of Hep-2 and AMC-HN-8 cells after HOXB9 knockout. In the growth curve, it can be observed that the OD value of Hep-2 cells in the HOXB9-ko group and NC control group at different culture time points $(48,72$ and 96 hours) are significantly different (Figure 3A). These results show that the knockout of HOXB9 expression in Hep-2 and AMC-HN-8 cells could delay the growth of LSCC cells, and inhibit the proliferation of LSCC cells. Next, flow cytometry was performed to detect the proportion of LSCC cells in the different phases. This can further reflect the effect of HOXB9 on the proliferation of LSCC cells. It was found that the cell cycle of Hep-2 and AMC-HN-8 cells in the experimental group significantly changed, when compared to the control group. Furthermore, the number of cells in the G1 phase was significantly increased, the $S$ phase was shortened, and the G2 phase had no significant changes (Figure 3B). These results show that the cell cycle of LSCC cells was blocked in the G1 phase after HOXB9 knockout. Furthermore, in order to investigate the effect of HOXB9 on the migration ability of LSCC cells, wound-healing assays were used to detect the effect of HOXB9 on the migration ability of LSCC cells. By comparing the images taken at 12 and 24 hours after cell migration, it was found that the migration ability of Hep-2 and AMC-HN-8 cells decreased in the HOXB9-KO group, when compared to the control group (Figure 3C). This suggests that HOXB9 can promote the migration of LSCC cells. Then, it the effect of HOXB9 on the invasiveness of LSCC cells was tested by Transwell assays. It was revealed that cell invasiveness decreased, when compared with the control group (Figure 3D). In addition, TUNEL assays were performed to determine whether HOXB9 affects LSCC cell apoptosis. The results revealed that after HOXB9 was knocked out in LSCC cells, the apoptosis of cells increased in the HOXB9-KO group, when compared to the control group (Figure 3E). 
These results suggest that HOXB9 could inhibit the apoptosis of LSCC cells. Furthermore, the mouse xenograft assays performed using HOXB9 knockout Hep-2 cells revealed that the tumor growth rate and tumor volumes significantly decreased in the HOXB9-KO group, when compared to the control group (Figure 3F). This indicates that HOXB9 can influence the proliferation ability of LSCC cells. Thus, these data suggests that HOXB9 can affect the cellular functional changes of LSCC cells.

Screening of differentially expressed genes after the knockout of HOXB9 in

\section{LSCC cells}

In order to investigate the downstream target gene regulated by HOXB9 in LSCC, the whole gene expression microarray was carried out after the CRISPR/Cas9-mediated deletion of HOXB9 in the Hep-2 cell line, and the significant differential expression genes were systematically screened out. The results revealed that there were 137 upregulated genes, 148 downregulated genes, and 21,614 genes without significant difference. The differences of these upregulated and downregulated genes are presented in three experimental groups and three control groups (Figure 4A). Next, a series of genes correlated to tumor cell proliferation, invasion and apoptosis was obtained by Gene ontology (GO) and Kyoto Encyclopedia of Genes and Genomes (KEGG) enrichment analysis, as shown in the typical gene thermogram (Figure 4B). A total of 10 representative tumor related genes were selected from these differentially expressed genes, and these were verified by RT-qPCR in Hep-2 cells with knocked out HOXB9 (three different biological repeats). The results revealed that the mRNA levels of these genes were consistent with the results of the ChIP assay (Figure $4 \mathbf{C}$ ). According to the differential gene screening 
experiment, MMP12 was noted to have the most significant difference, and this was also reported to be overexpressed in multiple tumors, and involved in the process of tumor growth, invasion and metastasis. In order to verify the correlation between the expression of HOXB9 and MMP12, the protein expression level of these two genes was detected in Hep-2 cells. The results revealed that the expression of MMP12 was downregulated when HOXB9 was knocked out (Figure 4D), and upregulated when HOXB9 was overexpressed (Figure 4E). These results show that the expression level of MMP12 changed with the HOXB9.

\section{The expression between HOXB9 and MMP12 is positively correlated}

In order to further clarify the correlation between the expression of HOXB9 and MMP12, the expression of HOXB9 and MMP12 was detected in 106 cases of LSCC and its adjacent tissues by immunohistochemistry. The results revealed that MMP12 was highly expressed in the cytoplasm of LSCC tissues, but lowly expressed in adjacent tissues (Figure 5A). The expression of MMP12 and HOXB9 have the same pattern in LSCC tissues (Figure 5B). According to Fisher's test, there was statistical significance in the correlation between the expression of HOXB9 and MMP12 (Figure 5C) $(P<0.001)$. The percentage stacking diagram further verified the correlation between the expression of HOXB9 and MMP12 in LSCC at different expression levels (Figure 5D). The final staining score was further calculated, and the correlation analysis curve was drawn according to the immunohistochemical staining scoring standard. It was found that as the HOXB9 expression in LSCC tissues increased, the expression of MMP12 also increased, showing a significantly positive correlation (Figure 5E), and the results were statistically significant (Pearson's statistical analysis, $P<0.0001)$. The Kaplan-Meier method was used to further 
compare the relationship between the expression level of HOXB9 and MMP12, and the prognosis of LSCC patients. These results revealed that the 5-year survival rate of patients was higher when these were both lowly expressed, and lower when these were both highly expressed (Figure 5F) $(P<0.01)$. These results show that the high expression of both HOXB9 and MMP12 could significantly reduce the 5-year survival rate of LSCC patients.

\section{HOXB9 can bind to the promoter of MMP12 and enhance its activity at the} transcription level

In order to determine whether HOXB9 activated the promoter of MMP12, a luciferase gene reporting plasmid of MMP12 3'-UTR was constructed (Figure 6A). Then, the Luciferase report plasmids were transfected into overexpressed HOXB9 and control cells, and knocked out HOXB9 and control Hep-2 cells. The Luciferase activities were detected in all groups. Each experimental group was designed with three multiple pores, and the experiment was repeated for three times. The Luciferase analysis revealed that compared with the pcDNA3.1 empty vector, the luciferase activity of MMP12 in wild-type HEK-293T cells that overexpressed HOXB9 significantly increased, and these results were statistically significant $(P<0.01)$, while the luciferase activity of MMP12 in mutant HEK-293T cells that overexpressed HOXB9 was not statistically different (Figure 6B). After HOXB9 was knocked out in Hep-2 cells, the luciferase activity of wild-type MMP12 significantly decreased, and the results were statistically significant $(P<0.001)$, while the luciferase activity of mutant MMP12 had no statistical difference (Figure 6C). These results show that HOXB9 could affect the activity of MMP12. In order to further verify whether the promoter region of the MMP12 gene can bind to HOXB9, the chromatin 
immunoprecipitation assay was used to determine whether HOXB9 can directly bind to the MMP12 promoter. The mouse IgG antibody was selected as the negative control, the anti-histone $\mathrm{H} 3$ antibody was selected as the positive control, and the anti-flag-HOXB9 antibody was selected as the experimental group. Then, the empty plasmid and HOXB9-flag overexpression plasmid were transferred in HEK-293T cells and Hep-2 cells, respectively. Finally, the purified DNA was obtained for RT-qPCR detection. These results revealed that the immunoprecipitation of the anti-flag-HOXB9 antibody in HEK-293T and Hep-2 significantly increased, and the results were statistically significant (Figures 6D and 6E, $P<0.001$ ). These results show that there was physical binding between the HOXB9 and MMP12 promoter.

\section{DISCUSSION}

LSCC is one of the most common head and neck cancers in the world. Despite the improvements in treatment over the past 10 years, the mortality rate remains high. ${ }^{19}$ The mechanism of LSCC remains unclear, and there is no effective treatment target. Furthermore, the occurrence and development of LSCC is a complex process, which involves the interaction of many factors. In the previous study conducted by the investigators, a group of HOX genes were screened out by microarray, which were upregulated in LSCC tissues. Further large sample microarray studies have shown that the high HOXB9 expression level is highly correlated with LSCC histological grade and poor prognosis. ${ }^{17}$ These results suggest that as a member of the HOX gene family, HOXB9 may be an effective biomarker for the development and prognosis of LSCC. However, it remains unclear how HOXB9 participates in LSCC.

In the present study, an attempt was made to elucidate the relationship between HOXB9 and LSCC progression in vivo and in vitro, and screen the downstream target 
gene of HOXB9, which is a transcription factor. Previous studies have revealed that HOXB9 is abnormally expressed in many tumors. However, this has different methods of action in different tumors. ${ }^{20-24}$ As it is known, the over proliferation of cells is a typical feature of cancer cells, and molecules correlated to the proliferation ability of different types of tumor cells play different roles. Researchers have reported that HOXB9 can promote the proliferation of tumor cells in different methods in breast cancer, liver cancer and colon cancer. ${ }^{25-27}$ These results are consistent with the present experimental results. In the present study, through the CCK-8 proliferation assay, it was observed that the LSCC cell lines knocked out of HOXB9 exhibited a significant decrease in proliferation ability. The subcutaneous tumorigenesis experiment in nude mice has further proven that HOXB9 can significantly promote the growth of LSCC transplanted tumors. However, the function of HOX family genes is complex, and the same gene can play an opposite role in different tumors. In a study on gastric cancer, it was found that HOXB9 can inhibit the proliferation of gastric cancer cells by inhibiting the expression of phosphorylated Akt and the activity of NF-kB. ${ }^{28}$ This completely opposite conclusion suggests that the role of HOXB9 in tumors is regulated by many factors, such as the cell genetic background.

The abnormal proliferation of tumor cells is closely correlated to the imbalance of the cell cycle and the resistance to apoptosis. In the present study, flow cytometry and TUNEL assays were performed to detect LSCC cells after the knockout of HOXB9. It was found that HOXB9 can significantly accelerate the cell cycle and inhibit tumor cell apoptosis. A similar study also revealed that in breast cancer, HOXB9 can be regulated by estrogen, thereby affecting the cell cycle process. This effect is achieved by binding HOXB9 with various tumor growth factor promoter regions, and regulating their transcription activity. ${ }^{29}$ In addition, researchers have 
found that in malignant melanoma, the HOXB9-dependent and microRNA765-mediated FOXA2 pathway can induce tumor stem cells to resist the apoptosis caused by exogenous stimulation, thereby maintaining the proportion of carcinoembryonic stem cells. ${ }^{30}$ However, the regulation of the HOX gene to the downstream gene is a complex network regulation. Therefore, further research is needed to explore these specific mechanisms.

The metastasis of malignant tumors is closely correlated to the significant enhancement of the ability of migration and invasion. In the present study, through scratch and Transwell experiments, it was observed that the migration and invasion ability of LSCC cell lines were markedly reduced after HOXB9 was knocked out. The histological types of oral squamous cell carcinoma (OSCC) and LSCC are very similar. In a study, it was found that HOXB9 can stimulate the migration and invasion of OSCC cells in vitro, and that this effect was induced through the activation of the TGF $31 /$ Smad2/slug signaling pathway, which caused the epithelial-to-mesenchymal transition (EMT), and increased the migration and invasion ability. ${ }^{31}$ Similar research results have also been reported in studies on endometrial cancer. Researchers have found that HOXB9 can regulate its activity by binding to the promoter region of E2F3. This direct target binding promotes the expression level of E2F3, and improves the invasion ability of endometrial cancer cells. ${ }^{32}$

The HOX gene is a member of the transcription factor family. Hence, its regulation of the target gene is often at the transcription level. At present, few studies have determined how the HOX gene regulates the target gene to promote tumor progression. It is very important to study the regulation of the HOX gene to the target gene, in order to understand the role of the HOX gene in tumor progression, and design an effective interference blocking target. In the present study, it was found that 
HOXB9 is highly expressed in LSCC, and that HOXB9 can affect the biological behavior of LSCC cells. In order to further investigate the specific mechanism, HOXB9 gene knockout was carried out on LSCC cells, and gene chip experiments were performed in the control group. Combined with bioinformatics tools, RT-qPCR, western blot, immunohistochemistry and other experiments, MMP12 was preliminarily screened as one of the downstream target genes regulated by HOXB9. Matrix metalloproteinase (MMP) is a zinc-dependent endopeptidase family. MMP is considered to be an important inflammatory mediator, which regulate tissue remodeling in physiological and pathological processes. ${ }^{33}$ MMPs have been proposed as a potential therapeutic target for various cardiovascular and musculoskeletal diseases and cancer. ${ }^{34}$ MMP12 belongs to the elastase family of MMP, and its function is mainly involved in the process of inflammation, tissue repair, respiratory diseases, and tumor growth, invasion and metastasis. ${ }^{35}$ Previous studies have reported that MMP12 is involved in the invasion and metastasis of non-small cell lung cancer (NSCLC). ${ }^{36}$ In addition, it was found that the expression of MMP12 is significantly elevated in lung adenocarcinoma tissues and cell lines, and that this is highly correlated with the pathological stage and lymph node metastasis. Furthermore, MMP12 knockout inhibits the proliferation and invasion of lung adenocarcinoma cells, suggesting that MMP12 may be a target for the treatment of lung adenocarcinoma. ${ }^{37}$ Some researchers have reported that the high level of MMP12 in serum is correlated to the poor prognosis of patients, suggesting that MMP12 may promote the invasion and metastasis of colon cancer cells. ${ }^{38}$ In the present study, it was speculated that MMP12 may be the downstream target gene of HOXB9, which is regulated by HOXB9 transcription, and has enhanced activity in LSCC, thereby affecting the 
biological behavior of LSCC cells.

In order to confirm the interaction between HOXB9 and MMP12, the chromatin immunoprecipitation and luciferase reporter gene assay were respectively performed. This confirmed the physical binding between the HOXB9 and MMP12 promoter, and revealed that HOXB9 can enhance the transcription activity of MMP12. Combined with the comparison of HOXB9 and MMP12 immunohistochemistry scores and patient survival time, it was found that the 5-year survival time in the HOXB9 and MMP12 co-overexpression group was significantly lower than in the other groups $(P=0.0031)$. The Pearson's analysis revealed that the expression of HOXB9 and MMP12 in LSCC was significantly positive correlated $(P<0.0001)$. All these above results suggest that $\mathrm{HOXB9}$ can regulate MMP12 at the transcription level, and promote the progression of LSCC.

The target gene of the HOX family transcription factor is very important to control cell biological behavior. To date, no specific target gene of HOXB9 has been identified. Furthermore, the regulation of HOXB9 on target genes requires the participation of some synergistic factors, such as PBX. In the present study, it was confirmed that HOXB9 can enhance the expression and activity of MMP12 at the transcription level. However, further studies are needed in the future to determine whether there are other synergistic factors involved in this regulation.

\section{CONCLUSION}

These results suggested that HOXB9 could play an important role in promoting cell proliferation, invasion and migration in LSCC via by enhancing the upregulation of MMP12. Importantly, HOXB9 promoted LSCC progression by upregulating MMP12 protein expression in LSCC cells via direct binding to the promoter of MMP12. 


\section{DECLARATIONS}

-Ethics approval and consent to participate

This study was approved by the Ethics Committee of Guizhou University of Traditional Chinese Medicine (K2020-006) and informed consent was obtained in accordance with ethical standards of the Declaration of Helsinki. All the animal experiments were performed in accordance with institutional guidelines and were approved by the animal care review board of Guizhou University of Traditional Chinese Medicine.

- Consent for publication

Not applicable.

- Availability of data and materials

The datasets during and/or analysed during the current study available from the corresponding author on reasonable request.

-Competing interests

The authors declare that they have no competing interests.

$\bullet$ Funding

This study was supported by the funds of the National Natural Science Foundation of China (Grant no. 82060495 to Chuanhui Sun and Grant no. 81272965,81772874 to Yanan Sun ).

- Authors' contributions

Chuanhui Sun and Peng Wang performed the cytological experiment. Yujiang Chen and Changsong Han were major contributors in animal and pathological experiment. Hua Deng and Qiuying Li performed the Immunohistochemical and western test. Yanan Sun was a major contributor in analyzing the data and writing the manuscript. All authors read and approved the final manuscript. 
-Acknowledgements

Not applicable

\section{REFERENCES}

1. Siegel RL, Miller KD, Jemal A. Cancer Statistics, 2017[J].CA Cancer J Clin,2017, 67(1):7-30.

2. Zhang S Y , Lu Z M , Luo X N , et al. Retrospective Analysis of Prognostic Factors in 205 Patients with Laryngeal Squamous Cell Carcinoma Who Underwent Surgical Treatment[J]. PLOS ONE, 2013, 8.

3. Bernstein J M , Clare R. Bernstein $\cdots$. Molecular and cellular processes underlying the hallmarks of head and neck cancer[J]. European Archives of Oto-Rhino-Laryngology, 2013, 270(10):2585-2593.

4. Garcia-Fernàndez, Jordi. The genesis and evolution of homeobox gene clusters[J]. Nature Reviews Genetics, 2005, 6(12):881-892.

5. Bürglin, Thomas R, Affolter M . Homeodomain proteins: an update[J]. Chromosoma, 2016, 125(3):497-521.

6. Barber B A, Rastegar M . Epigenetic control of Hox genes during neurogenesis, development, and disease.[J]. Annals of Anatomy, 2010, 192(5):261-274.

7. Soshnikova N . Hox genes regulation in vertebrates[J]. Developmental Dynamics, 2014, 243(1).

8. Carrio M, Arderiu G, Myers C, et al. Homeobox D10 Induces Phenotypic Reversion of Breast Tumor Cells in a Three-Dimensional Culture Model[J]. Cancer Research, 2005, 65(16):7177-7185.

9. Taketani T , Taki T, Shibuya N , et al. Novel NUP98-HOXC11 fusion gene resulted from a chromosomal break within exon 1 of HOXC11 in acute myeloid leukemia with $\mathrm{t}(11 ; 12)(\mathrm{p} 15 ; \mathrm{q} 13)[\mathrm{J}]$. Cancer Research, 2002, 62(16):4571-4574.

10. Nguyen D X , Chiang A C , Zhang H F , et al. WNT/TCF Signaling through LEF1 and HOXB9 Mediates Lung Adenocarcinoma Metastasis[J]. Cell, 2009, 138(1):0-62.

11. Plowright L, Harrington K J , Pandha H S , et al. HOX transcription factors are potential therapeutic targets in non-small-cell lung cancer (targeting HOX genes in lung cancer)[J]. British Journal of Cancer, 2009, 100(3):470-475.

12. Tsai K W , Liao Y L, Wu C W, et al. Aberrant expression of miR-196a in gastric cancers and correlation with recurrence[J]. Genes, Chromosomes and Cancer, 2012, 51(4):394-401.

13. Shimoda, M, Takahashi, M, Yoshimoto, T, et al. A Homeobox Protein, Prox1, Is Involved in the Differentiation, Proliferation, and Prognosis in Hepatocellular Carcinoma[J]. Clinical Cancer Research, 2006,12(20):6005-6011.

14. Hayashida T, Takahashi F, Chiba N, et al. HOXB9, a gene overexpressed in breast cancer, promotes tumorigenicity and lung metastasis[J]. Proceedings of the National Academy of Sciences of the United States of America, 2010, 107(3):1100-1105.

15. Shrestha B , Ansari K I, Bhan A, et al. Homeodomain-containing protein HOXB9 regulates expression of growth and angiogenic factors, facilitates tumor growth in?vitro and is overexpressed in breast cancer tissue[J]. Febs Journal, 2012, 279(19).

16. Chiba N, Comaills V, Shiotani B , et al. Homeobox B9 induces epithelial-to-mesenchymal transition-associated radioresistance by accelerating DNA damage responses[J]. Proceedings 
of the National Academy of Sciences of the United States of America, 2012, 109(8):2760-2765.

17. Chuanhui S , Changsong H, Peng W , et al. HOXB9 Expression Correlates with Histological Grade and Prognosis in LSCC[J]. BioMed Research International, 2017, 2017:1-10.

18. Peng W, Tianyi W, Han Z, et al. Long noncoding RNA NEAT1 promotes laryngeal squamous cell cancer through regulating miR-107/CDK6 pathway[J]. Journal of Experimental \& Clinical Cancer Research, 2016, 35(1):22.

19. Jou A , Hess J . Epidemiology and Molecular Biology of Head and Neck Cancer[J]. Oncology Research and Treatment, 2017, 40(6):328-332.

20. Vychytilova-Faltejskova P, Merhautova J , Machackova T, et al. MiR-215-5p is a tumor suppressor in colorectal cancer targeting EGFR ligand epiregulin and its transcriptional inducer HOXB9[J]. Oncogenesis, 2017, 6(11):5.

21. Deb P, Bhan A, Hussain I, et al. Endocrine disrupting chemical, bisphenol-A, induces breast cancer associated gene HOXB9 expression in vitro and in vivo[J]. Gene, 2016: 590(2):234-243.

22. Kwon O S , Oh E, Park J R , et al. GalNAc-T14 promotes metastasis through Wnt dependent HOXB9 expression in lung adenocarcinoma[J]. Oncotarget, 2015, 6(39):41916-41928.

23. Fang L, Xu Y, Zou L. Overexpressed homeobox B9 regulates oncogenic activities by transforming growth factor- $\beta 1$ in gliomas[J]. Biochemical and Biophysical Research Communications, 2014, 446(1):272-279.

24. Chang Q, Zhang L, He C , et al. HOXB9 induction of mesenchymal-to-epithelial transition in gastric carcinoma is negatively regulated by its hexapeptide motif[J]. Oncotarget, 2015, 6(40):42838-42853.

25. Seki H1, Hayashida T, Jinno H, Hirose S, Sakata M, Takahashi M, Maheswaran S, Mukai M, Kitagawa Y. HOXB9 expression promoting tumor cell proliferation and angiogenesis is associated with clinical outcomes in breast cancer patients[J]. Ann Surg Oncol,2012,19(6):1831-1840.

26. Li F , Dong L, Xing R, et al. Homeobox B9 is overexpressed in hepatocellular carcinomas and promotes tumor cell proliferation both in vitro and in vivo[J]. Biochemical and Biophysical Research Communications, 2014, 444(2):241-247.

27. Hoshino Y , Hayashida T , Hirata A, et al. Bevacizumab terminates homeobox B9-induced tumor proliferation by silencing microenvironmental communication[J]. Molecular Cancer, 2014, 13(1):102.

28. Li, Zhang, Qinghua, et al. HOXB9 inhibits proliferation in gastric carcinoma cells via suppression of phosphorylated-Akt and NF-KB-dependent Snail expression.[J]. Dig Liver Dis, 2019,51(1):157-165.

29. Shrestha B , Ansari K I, Bhan A, et al. Homeodomain-containing protein HOXB9 regulates expression of growth and angiogenic factors, facilitates tumor growth in?vitro and is overexpressed in breast cancer tissue[J]. Febs Journal, 2012, 279(19).

30. Lin J , Zhang D, Fan Y, et al. Regulation of cancer stem cell self-renewal by HOXB9 antagonizes ER stress-induced melanoma cell apoptosis via the miR-765-FOXA2 axis[J]. J Invest Dermatol,2018;138 (7):1609-1619.

31. Xue M, Zhu F Y, Chen L, et al. HoxB9 promotes the migration and invasion via TGF- $\beta$ 1/Smad2/Slug signaling pathway in oral squamous cell carcinoma[J]. Am J Transl Res, 2017, 
9(3):1151-1161.

32. Wan J , Liu H, Feng Q , et al. HOXB9 promotes endometrial cancer progression by targeting E2F3[J]. Cell death \& disease, 2018, 9(5):509.

33. Chen Q, Jin M, Yang F , et al. Matrix Metalloproteinases: Inflammatory Regulators of Cell Behaviors in Vascular Formation and Remodeling[J]. Mediators of Inflammation, 2013, 2013:1-14.

34. Cui N, Hu M, Khalil R A . Biochemical and Biological Attributes of Matrix Metalloproteinases[J]. Prog Mol Biol Transl Sci,2017,147:1-73. .

35. Velinov N, Poptodorov G, Gabrovski N, et al. The role of matrix metalloproteinases in the tumor growth and metastasis[J]. Khirurgiia, 2010, 1(1):44-49.

36. Wen Y, Cai L . Research Progress of Matrix Metalloproteinase 12 in Non-small Cell Lung Cancer[J]. Zhongguo Fei Ai Za Zhi,2014, 17(1):30-33.

37. Lv F, Wang J, Wu Y, et al. Knockdown of MMP12 inhibits the growth and invasion of lung adenocarcinoma cells[J]. International Journal of Immunopathology and Pharmacology, 2015, 28(1):77-84.

38. Klupp F, Neumann L, Kahlert C, et al. Serum MMP7, MMP10 and MMP12 level as negative prognostic markers in colon cancer patients[J]. BMC Cancer,2016,16:494. 


\section{FIGURE LEGENDS}

Figure 1 Differences in the expression of HOXB9 in the LSCC cell lines. (A) The RT-qPCR revealed that the expression of HOXB9 in Hep-2 and AMC-HN-8 cells was significantly higher than that in $\mathrm{HaCaT}, * * * P<0.001$; (B) The western blot results revealed that the expression of HOXB9 in Hep-2 and AMC-HN-8 cells was significantly higher than that in HaCaT.

Figure 2 Validation of the efficiency of HOXB9 knockout in LSCC cells. Western blot was used to detect the protein expression in Hep-2 and AMC-HN-8 cells. Control: blank control; NC: Negative control sgRNA; KO-1-KO-3: HOXB9 sgRNA.

Figure 3 HOXB9 affects the cellular functional changes of LSCC cells. (A) The effect of HOXB9 knockout on the proliferation of LSCC cells. The OD values of NC and HOXB9-KO in Hep-2 cells at 24, 48, 72 and 96 hours were significantly different, The OD values of NC and HOXB9-KO in AMC-HN-8 cells at 24 hours were not significantly different, but the OD values at 48, 72 and 96 hours were significantly different $(* P<0.05, * * P<0.01$ and $* * * P<0.001)$. (B) Flow cytometry was used to detect the effect of HOXB9 on the LSCC cell cycle. Changes in the Hep-2 and AMC-HN-8 cell cycle after HOXB9 knockout are shown $(* * P<0.01)$. (C) Scratch assay was used to detect the effect of HOXB9 on the migration of LSCC cells. The difference in Hep-2 and AMC-HN-8 cells at 0, 12 and 24 hours between the experimental group and control group are shown, which were statistically significant $(* P<0.05$ and $* * P<0.01)$. Image magnification at $200 \times$. (D) Transwell assay was used to detect the effect of HOXB9 on the invasion of LSCC cells. The invasive ability of Hep-2 and AMC-HN-8 cells after HOXB9 knockout is shown, which was statistically significant $(* * P<0.01)$. Image magnification at $200 \times$. (E) TUNEL assay was used to 
detect the apoptosis of LSCC cells after HOXB9 knockout. The apoptotic expression of Hep-2 and AMC-HN-8 cells after HOXB9 knockout is shown. The first column of red fluorescence represents the apoptotic cells, the second column of blue fluorescence represents the nucleus, and the third column of pink fluorescence represents the merge of these two images. The magnification was 200×. (F) The effect of HOXB9 on the growth rate and size of LSCC subcutaneous tumors in nude mice is shown. The subcutaneous tumorigenesis of nude mice in the experimental and control groups are shown, and there was statistical significance in the tumor volume and weight between the experimental and control groups at 7, 10, 13, 16, 19, 22 and 25 days $(* * P<0.01$ and $* * * P<0.001)$.

Figure 4 The screening of differentially expressed genes after the knockout of HOXB9 in LSCC cells. (A) The microarray analysis of differentially expressed genes in Hep-2 cells after HOXB9 knockout. The red dots represent the upregulated genes, the green dots represent downregulated genes, and the gray dots represent the non-differentially expressed genes. Columns 1-6 represent the HOXB9 knockout (three biological repeats) and control group (three biological repeats), respectively. The change from green to red color indicates that the fold change is from low to high. (B) A typical gene thermogram is shown. The thermogram shows the hierarchical clustering, in which columns 1-6 represent the HOXB9 knockout group and control group, respectively. The change from green to red color indicates that the fold change is from low to high. (C) The RT-qPCR validation of the representative gene mRNA level is presented. The black columnar represents the result of the DNA microarray, while the white columnar represents the result of the RT-qPCR. It can be observed that these two results are basically the same. (D) The expression of MMP12 and HOXB9 in the knockout group and control group. (E) The expression of MMP12 and 
HOXB9 in the overexpression group and control group.

Figure 5 (A) The expression of MMP12 in LSCC and its adjacent tissues. The first row presents the expression of MMP12 in cancer tissues, and the second row presents the expression of MMP12 in adjacent tissues. The magnification was at $100 \times, 200 \times$ and 400×, respectively. (B) The expression of HOXB9 and MMP12 in LSCC tissues. The first and second rows represent the low expression of these two in cancer tissues, while the third and fourth rows represent the high expression of these two in cancer tissues. HOXB9 was brown-yellow in the nucleus, while MMP12 was brown-yellow in the cytoplasm. The magnification was at $100 \times, 200 \times$ and $400 \times$, respectively. (C) The Fisher's test revealed the correlation between these two in cancer tissues $(P<0.001)$. (D) The percentage stack chart revealed the correlation between these two in cancer tissues at different expression levels. (E) The correlation analysis of HOXB9 and MMP12 in LSCC tissues is shown. The Pearson's statistical analysis revealed the correlation between the HOXB9 and MMP12 staining scores $(P<0.0001)$. (F) The expression of HOXB9 and MMP12 was compared with the survival rate of LSCC patients. Both of these were lowly expressed, which is shown by the green line, and highly expressed, which is shown by the red line (Log rank statistical analysis, $P<0.01)$

Figure 6 (A) Schematic map of the 3'-UTR terminal binding sites of the wild and mutant types of HOXB9 in MMP12. WT: wild type, MT: mutant type. (B) The overexpression of HOXB9 in the 3'-UTR region of wild-type MMP12 significantly enhanced the luciferase activity $(* P<0.05)$. (C) The knockout of HOXB9 during the transfection of the wild-type 3'-UTR region of MMP12 significantly decreased the luciferase activity $(* * * P<0.001)$. (D) The RT-qPCR results of negative and positive in the control group and experimental group for the transfected empty plasmid and 
HOXB9-Flag overexpression plasmid in HEK-293T cells $(* * * P<0.001)$ and $(\mathrm{E})$ Hep-2 cells $(* * * P<0.001)$. Anti-IgG: mouse IgG antibody coprecipitation complex; Anti-Histone: anti-histone H3 antibody coprecipitation complex; Anti-Flag: anti-Flag antibody coprecipitation complex; Vector: transfected empty plasmid group; Flag-HOXB9: transfected HOXB9-Flag overexpression plasmid group. 


\section{Figures}

A

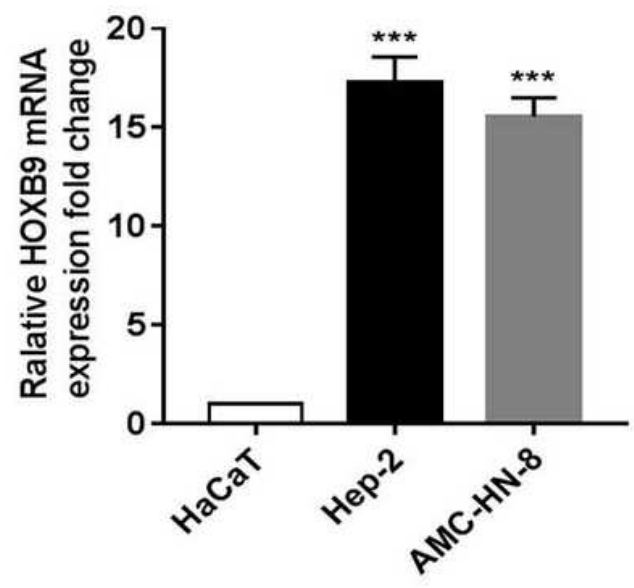

B

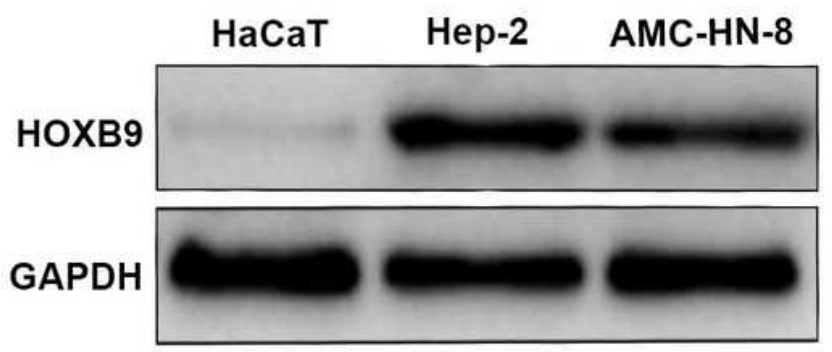

\section{Figure 1}

Differences in the expression of HOXB9 in the LSCC cell lines. (A) The RT-qPCR revealed that the expression of HOXB9 in Hep-2 and AMC-HN-8 cells was significantly higher than that in HaCaT, $\star \star \star P<0.001$; (B) The western blot results revealed that the expression of HOXB9 in Hep-2 and AMC-HN-8 cells was significantly higher than that in $\mathrm{HaCaT}$.

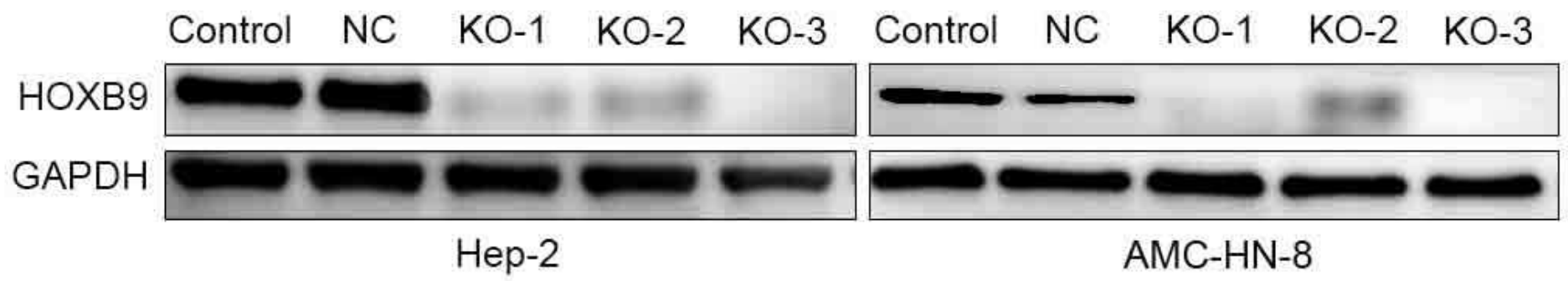

Figure 2

Validation of the efficiency of HOXB9 knockout in LSCC cells. Western blot was used to detect the protein expression in Hep-2 and AMC-HN-8 cells. Control: blank control; NC: Negative control sgRNA; KO-1-KO-3: HOXB9 sgRNA. 
A
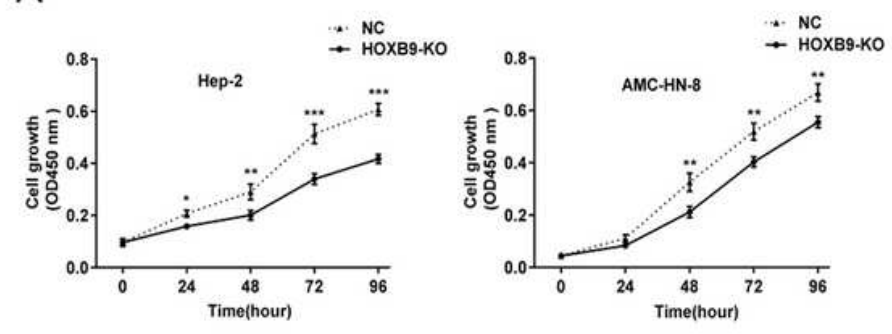

C
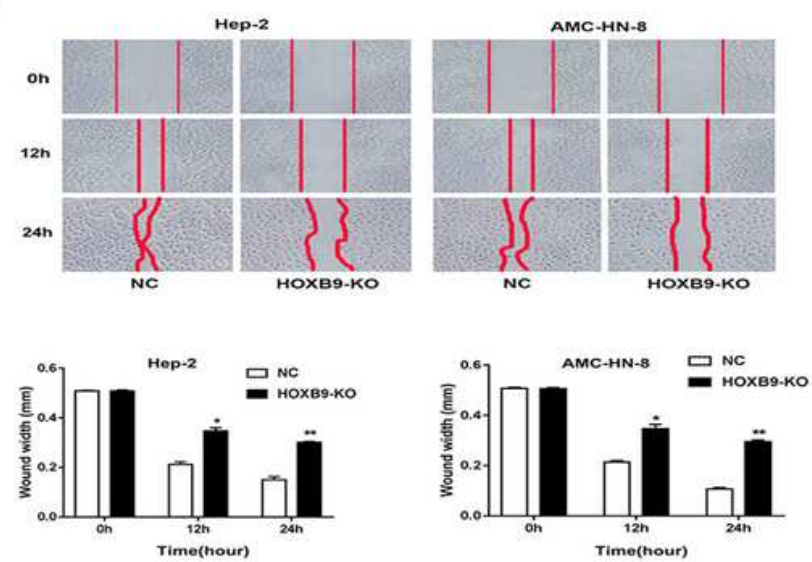

E

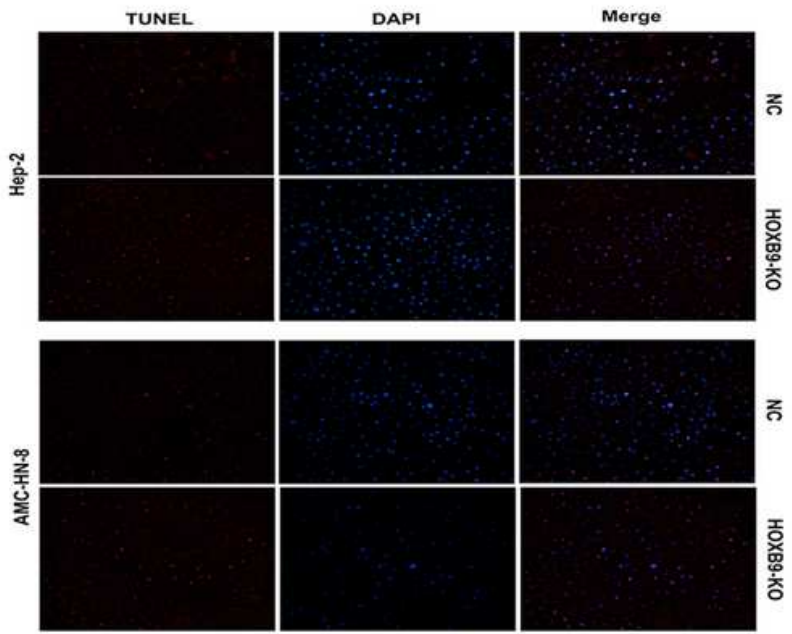

B
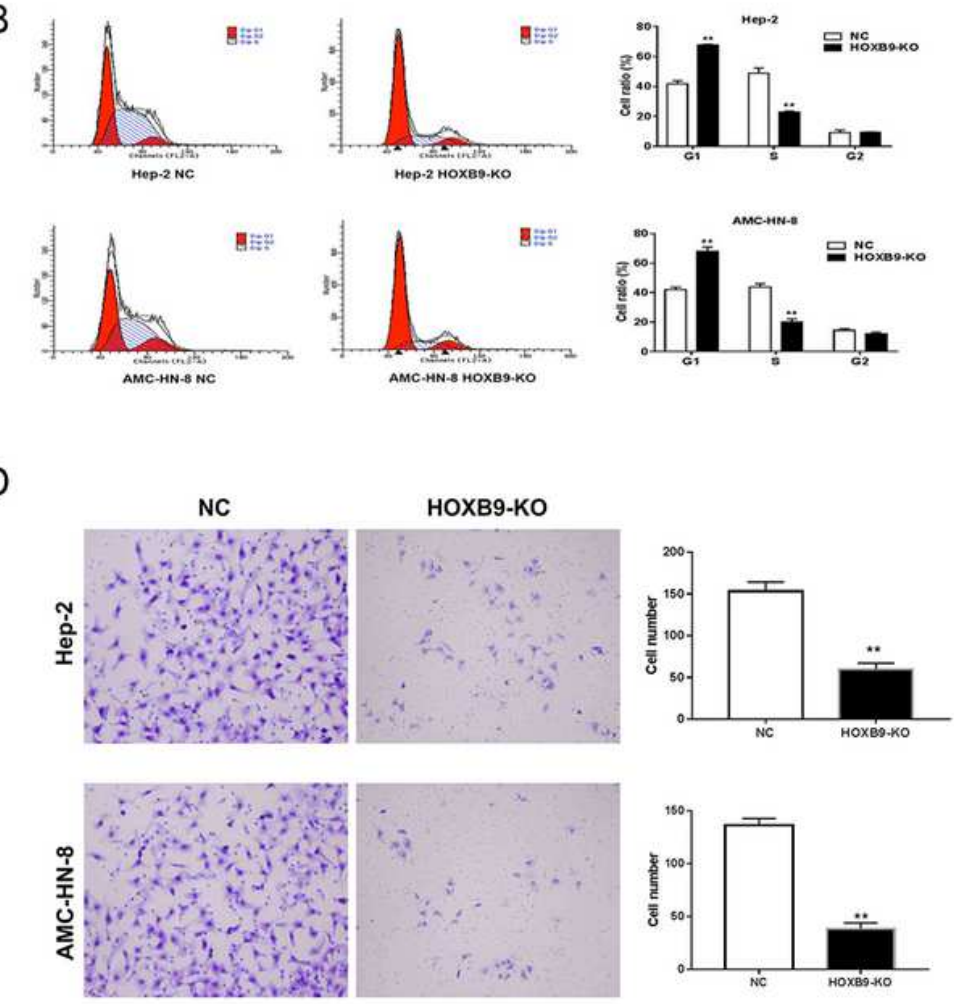

F
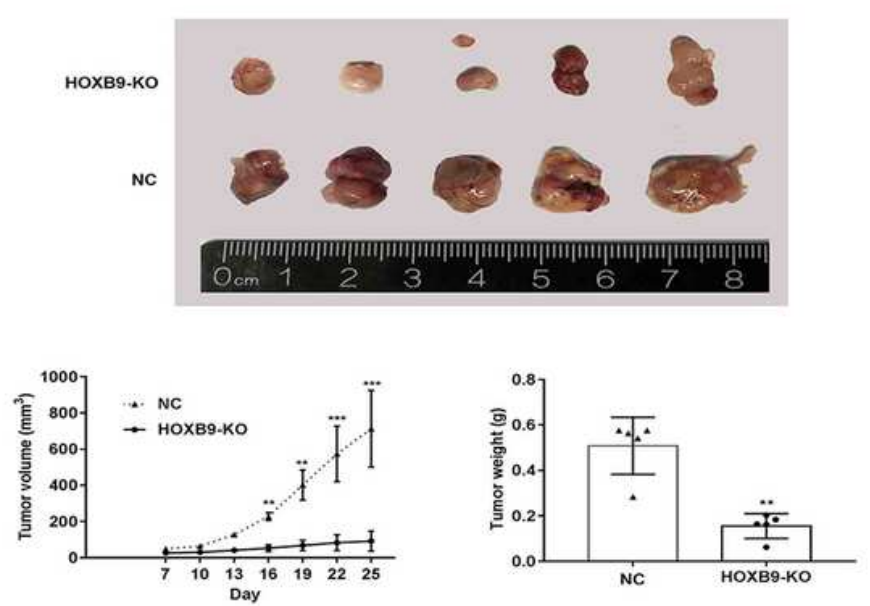

\section{Figure 3}

HOXB9 affects the cellular functional changes of LSCC cells. (A) The effect of HOXB9 knockout on the proliferation of LSCC cells. The OD values of NC and HOXB9-KO in Hep-2 cells at 24, 48, 72 and 96 hours were significantly different, The OD values of NC and HOXB9-KO in AMC-HN-8 cells at 24 hours were not significantly different, but the OD values at 48, 72 and 96 hours were significantly different $\left({ }^{\star} P<0.05\right.$, ${ }^{*} \mathrm{P}<0.01$ and $\left.* \star * P<0.001\right)$. (B) Flow cytometry was used to detect the effect of HOXB9 on the LSCC cell cycle. Changes in the Hep-2 and AMC-HN-8 cell cycle after HOXB9 knockout are shown (**P<0.01). (C) Scratch assay was used to detect the effect of HOXB9 on the migration of LSCC cells. The difference in Hep-2 and AMC-HN-8 cells at 0, 12 and 24 hours between the experimental group and control group are shown, which were statistically significant $\left({ }^{*} P<0.05\right.$ and $\left.{ }^{*} \mathrm{P}<0.01\right)$. Image magnification at $200 \times$. (D) 
Transwell assay was used to detect the effect of HOXB9 on the invasion of LSCC cells. The invasive ability of Hep-2 and AMC-HN-8 cells after HOXB9 knockout is shown, which was statistically significant (** $\mathrm{P}<0.01)$. Image magnification at 200x. (E) TUNEL assay was used to detect the apoptosis of LSCC cells after HOXB9 knockout. The apoptotic expression of Hep-2 and AMC-HN-8 cells after HOXB9 knockout is shown. The first column of red fluorescence represents the apoptotic cells, the second column of blue fluorescence represents the nucleus, and the third column of pink fluorescence represents the merge of these two images. The magnification was 200x. (F) The effect of HOXB9 on the growth rate and size of LSCC subcutaneous tumors in nude mice is shown. The subcutaneous tumorigenesis of nude mice in the experimental and control groups are shown, and there was statistical significance in the tumor volume and weight between the experimental and control groups at 7, 10, 13, 16, 19, 22 and 25 days $(* * P<0.01$ and $* * * P<0.001)$.

A
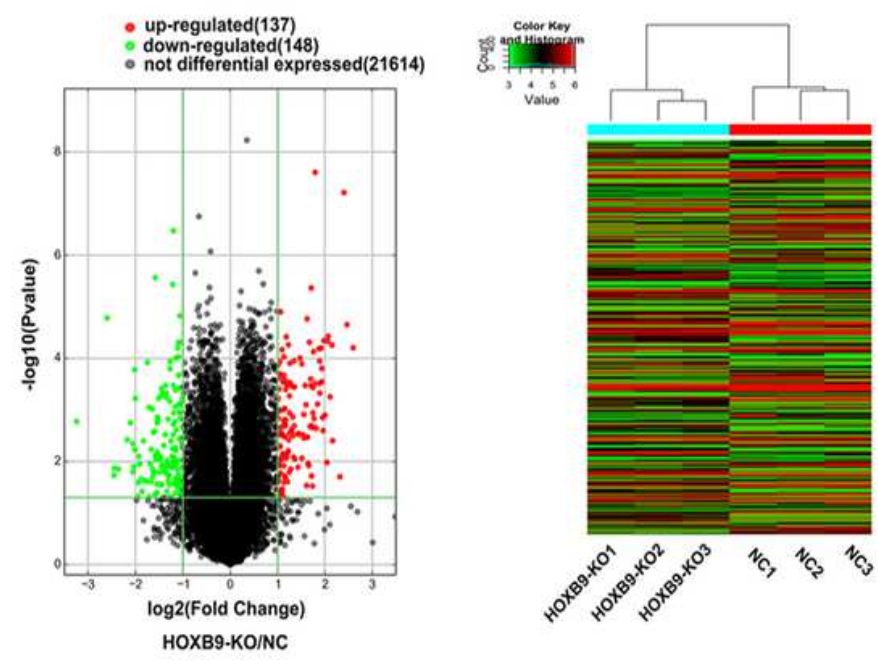

C

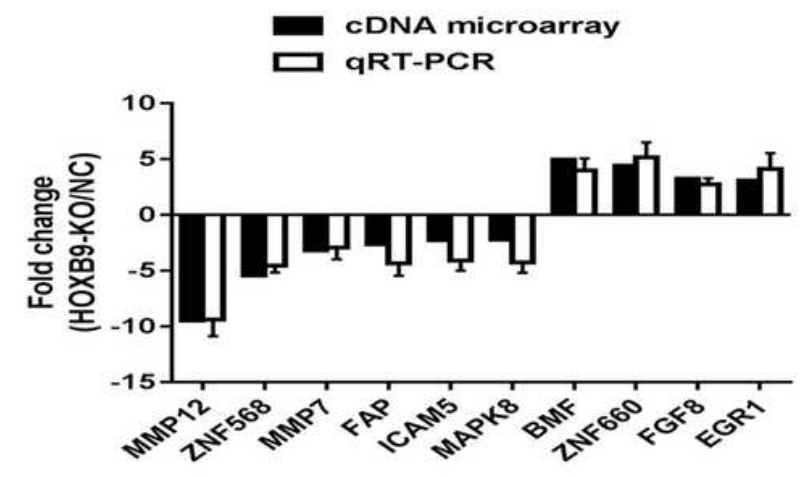

B

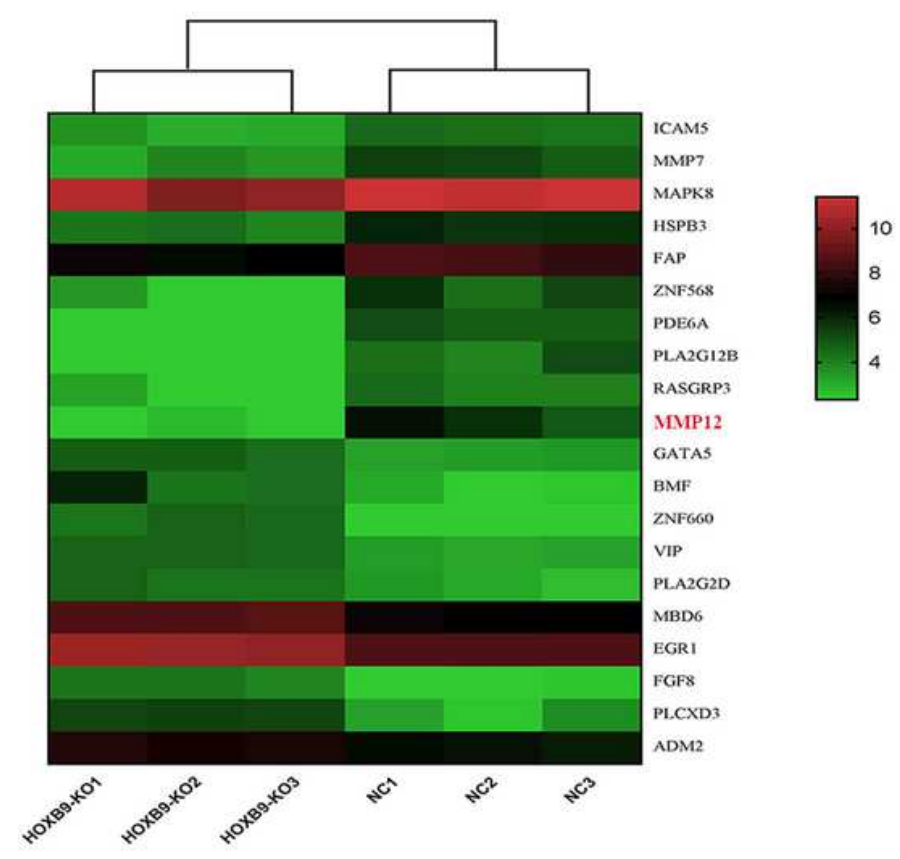

D

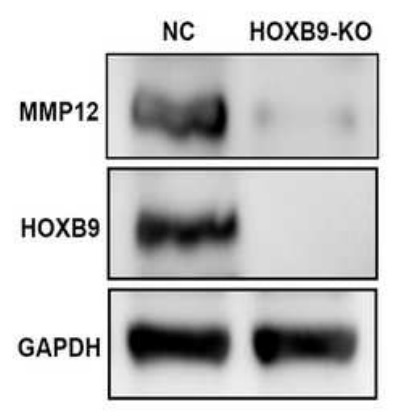

E

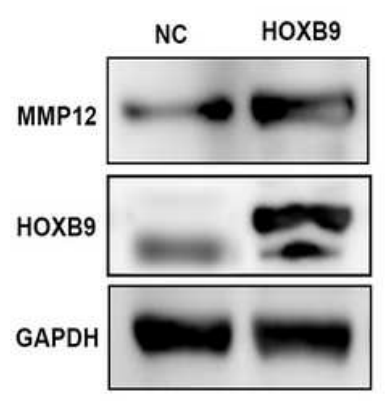

\section{Figure 4}


The screening of differentially expressed genes after the knockout of HOXB9 in LSCC cells. (A) The microarray analysis of differentially expressed genes in Hep-2 cells after HOXB9 knockout. The red dots represent the upregulated genes, the green dots represent downregulated genes, and the gray dots represent the non-differentially expressed genes. Columns 1-6 represent the HOXB9 knockout (three biological repeats) and control group (three biological repeats), respectively. The change from green to red color indicates that the fold change is from low to high. (B) A typical gene thermogram is shown. The thermogram shows the hierarchical clustering, in which columns 1-6 represent the HOXB9 knockout group and control group, respectively. The change from green to red color indicates that the fold change is from low to high. (C) The RT-qPCR validation of the representative gene mRNA level is presented. The black columnar represents the result of the DNA microarray, while the white columnar represents the result of the RT-qPCR. It can be observed that these two results are basically the same. (D) The expression of MMP12 and HOXB9 in the knockout group and control group. (E) The expression of MMP12 and HOXB9 in the overexpression group and control group.

A
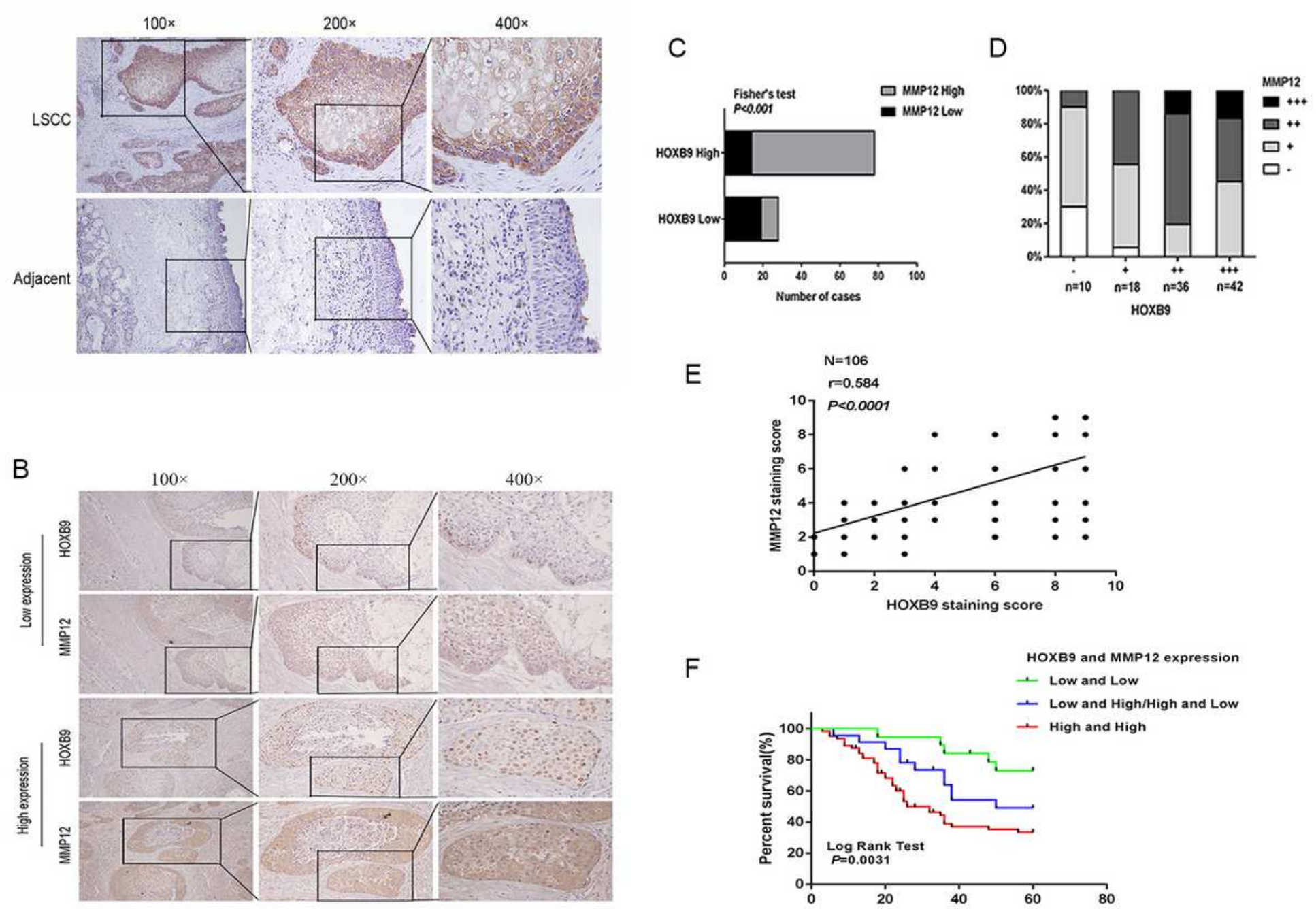

$\mathrm{F}$

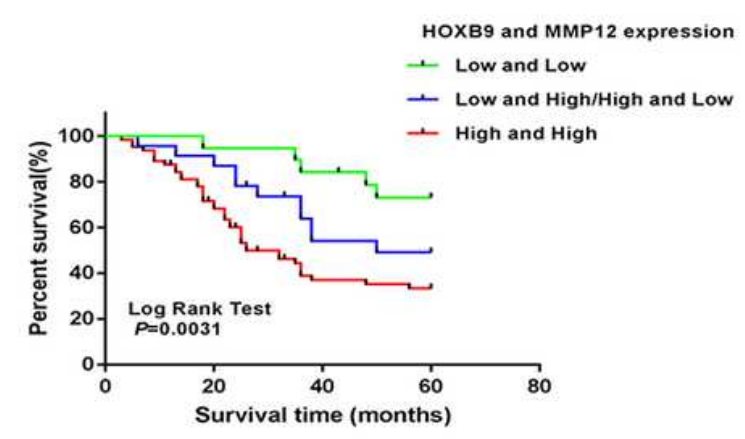

Figure 5 
(A) The expression of MMP12 in LSCC and its adjacent tissues. The first row presents the expression of MMP12 in cancer tissues, and the second row presents the expression of MMP12 in adjacent tissues. The magnification was at 100x, 200x and 400x, respectively. (B) The expression of HOXB9 and MMP12 in LSCC tissues. The first and second rows represent the low expression of these two in cancer tissues, while the third and fourth rows represent the high expression of these two in cancer tissues. HOXB9 was brown-yellow in the nucleus, while MMP12 was brown-yellow in the cytoplasm. The magnification was at 100x, 200xand 400x, respectively. (C) The Fisher's test revealed the correlation between these two in cancer tissues $(P<0.001)$. (D) The percentage stack chart revealed the correlation between these two in cancer tissues at different expression levels. (E) The correlation analysis of HOXB9 and MMP12 in LSCC tissues is shown. The Pearson's statistical analysis revealed the correlation between the HOXB9 and MMP12 staining scores $(P<0.0001)$. $(F)$ The expression of HOXB9 and MMP12 was compared with the survival rate of LSCC patients. Both of these were lowly expressed, which is shown by the green line, and highly expressed, which is shown by the red line (Log rank statistical analysis, $\mathrm{P}<0.01$ ). 
A

WT MMP12 3'UTR 5'...TTCAGTCC CTTA TTTT AAGGT...3'

HOXB9 3'...GCTACAGGGAATAAAA TTCCC...5'

MT MMP12 3'UTR 5'...TTCAAGGGCCCCCCGATCCCT....'

B

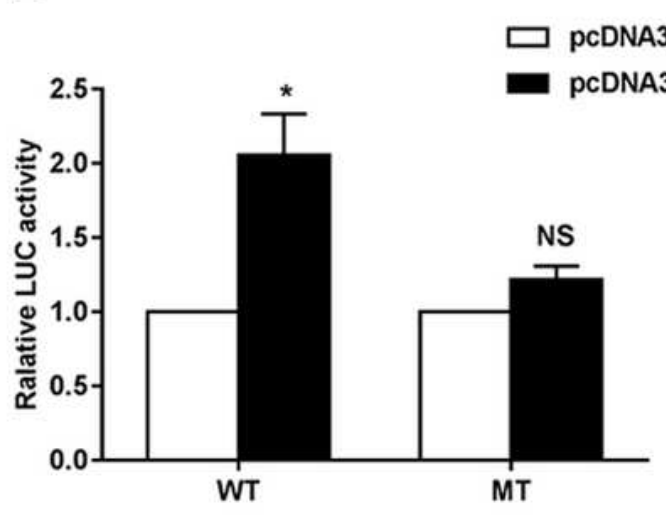

D

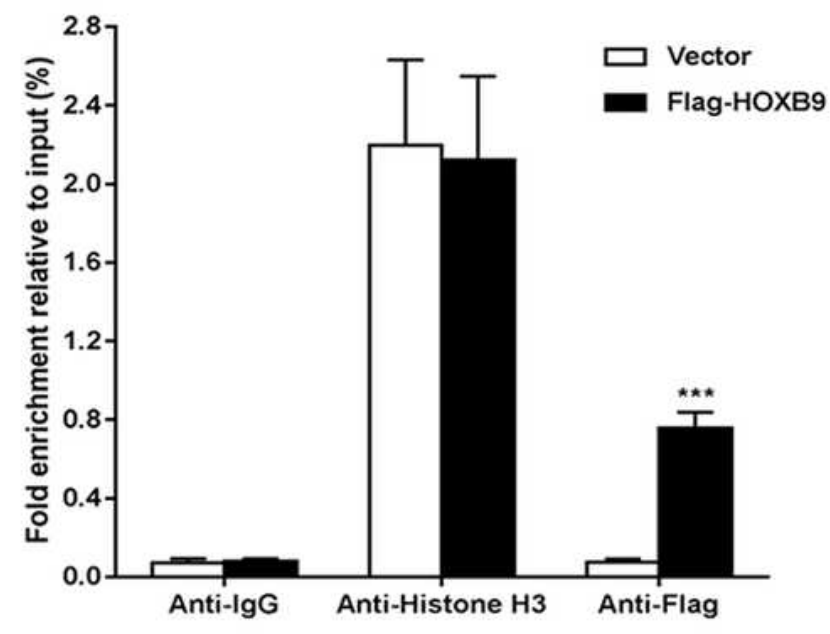

C

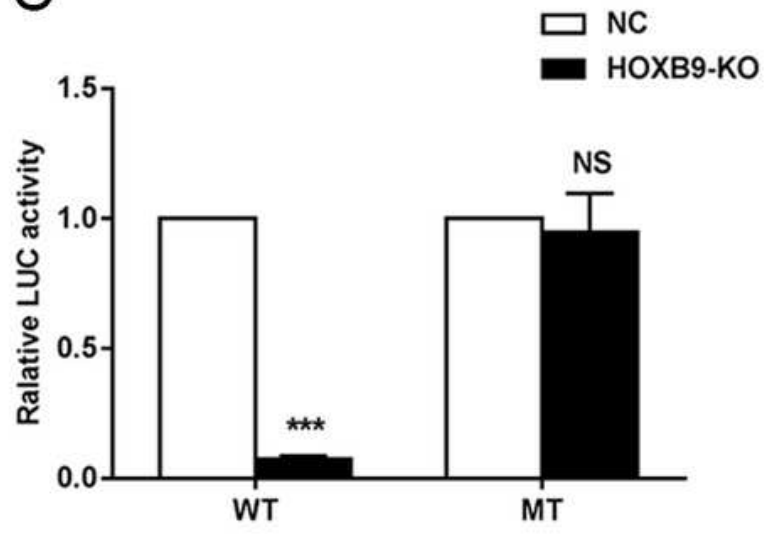

$E$

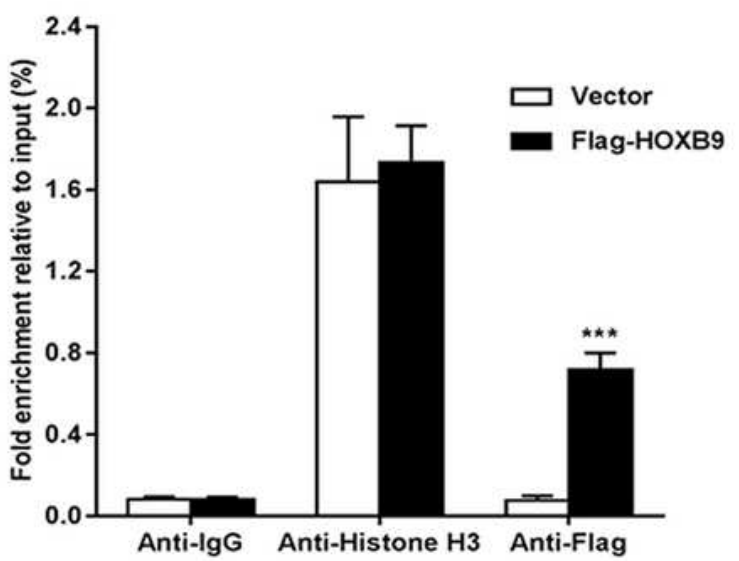

Figure 6

(A) Schematic map of the 3'-UTR terminal binding sites of the wild and mutant types of HOXB9 in MMP12. WT: wild type, MT: mutant type. (B) The overexpression of HOXB9 in the 3'-UTR region of wildtype MMP12 significantly enhanced the luciferase activity $\left({ }^{\star} P<0.05\right)$. (C) The knockout of HOXB9 during the transfection of the wild-type 3'-UTR region of MMP12 significantly decreased the luciferase activity ( $\left.{ }^{*} * P<0.001\right)$. (D) The RT-qPCR results of negative and positive in the control group and experimental 
group for the transfected empty plasmid and HOXB9-Flag overexpression plasmid in HEK-293T cells $(\star \star \star \mathrm{P}<0.001)$ and $(\mathrm{E}) \mathrm{Hep}-2$ cells $(\star \star \star \mathrm{P}<0.001)$. Anti-lgG: mouse IgG antibody coprecipitation complex; Anti-Histone: anti-histone $\mathrm{H} 3$ antibody coprecipitation complex; Anti-Flag: anti-Flag antibody coprecipitation complex; Vector: transfected empty plasmid group; Flag-HOXB9: transfected HOXB9-Flag overexpression plasmid group 